\title{
Topological Anomalies: Explicit Examples
}

\author{
Lay Nam Chang and Yigao Liang \\ Department of Physics, Virginia Polytechnic Institute and State University, Blacksburg, \\ VA 24061, USA
}

\begin{abstract}
We discuss the mathematical picture of anomalies. By solving the Dirac equation in the background of non-trivial families of gauge connections, we show explicitly the interplay between spectral flows, zero modes of the Dirac operator and projective representations of the gauge group, and the existence of both perturbative and non-perturbative anomalies. We give an explicit expression for the fermion determinant for chiral QCD in two dimensions when an anomaly is present.
\end{abstract}

\section{Introduction}

In recent years, considerable progress has been made in understanding the structure of the anomalies and their topological origin. By exploiting the cohomological properties $[1,2]$ of the Wess-Zumino consistent condition, one can derive the descent equations, and using these, a straightforward way of writing down gauge anomalies can be prescribed. Finally, one can give topological interpretations to these essentially algebraic constructions [3]. The mathematical foundations for anomalies were reviewed by Atiyah and Singer, and discussed within the framework of the family index theorem of elliptic operators [4].

However, the physical implications of the anomalies remain largely to be explored. As is well known, anomalies pose serious problems for the quantization of chiral fermions in gauge theories. In building models of elementary particles, the current wisdom is that anomalies are inconsistencies in the theory and have to be cancelled, giving rise to severe constraints on the resultant particle content. What other kinds of constraints do anomalies imply? It is, of course, far from clear that current methods of handling quantum field theories are adequate in the presence of anomalies, and if not, the structure of the anomalies may help in pointing out alternatives. In addition, there is also the question that even if anomalies are cancelled, what role each individual anomaly plays in the theory, especially in models of composite fermions. To further explore these questions, it is useful to examine more closely the structure of anomalies in the form of explicit solutions in 
theories which carry anomalies. This paper is a step in this direction. We show explicitly for representative field theories, how the existence of special solutions to the Dirac equation gives rise to ambiguities in the usual quantization procedure, thereby generating the anomalies. Our results cover the non-perturbative cases as well, where no local expressions for the anomalies exist. The examples we discuss substantiate the general topological arguments already given in the literature.

The plan of the paper is as follows. In Sect. 2, we review the geometrical setting and what is generally known about gauge theories and anomalies, and indicate how our results fit into this framework. In subsequent sections we study anomalies in simple but representative theories, which allow for explicit calculations, and which also have the requisite topological properties. In Sect. 3, we determine the eigenvalues of the Dirac operator on a spherical surface with a non-trivial family of $S U$ (2) background gauge fields, and obtain an exact expression for the chiral fermion determinant. The presence of unpaired zero modes is seen to produce a phase ambiguity in the determinant, which is akin to that in wave-functions around magnetic poles. In Sect. 4, similar results are obtained in the Hamiltonian approach for the same model. Here, it is the flow of an integral topological index that determines the anomaly. The fermionic states form a non-trivial bundle on the group of local gauge transformations. The Hamiltonian approach is then used in Sect. 5 to discuss the non-perturbative anomaly in $2+1$ dimensions. We demonstrate the fermionic states now form a non-orientable space on the group of local gauge transformations. The situation arises because of the flow of a $\mathbb{Z} / 2$ index associated to the energy levels. Section 6 contains some concluding remarks.

\section{Review of Known Results}

In $n$ dimensional Euclidean space-time, gauge fields with appropriate asymptotic behavior can be regarded to be on an $n$ dimensional sphere $S^{n}$. The gauge potentials are the connections of some principal bundle with a certain structure group, the gauge group. One usually associates the principal bundle with vector bundles through representations of the gauge group. The sections of the vector bundles tensoring with some spin bundles are called the matter fields. It can happen that there are many non-isomorphic bundles on $S^{n}$. Each isomorphism class is then called an instanton sector. The sectors are classified by $\pi_{n-1}(G)$, where $G$ is the gauge group. For each sector, the connections form an affine space $\mathbf{A}$. The gauge transformation group (the group of base preserving automorphisms of the principal bundle) acts on $\mathbf{A}$. The subgroup $\mathbf{G}$ that consists of those elements which leave the points above a base point (the north pole, say) in the space-time manifold fixed acts on $\mathbf{A}$ without fixed points $[5,6]$. Hence $P: \mathbf{A} \rightarrow \mathbf{A} / \mathbf{G}$ is a principal bundle. The gauge orbit space $\mathbf{A} / \mathbf{G}$ is therefore a classifying space of $\mathbf{G}$ because $\mathbf{A}$ is contractible. Also known is that $\mathbf{G}$ has the homotopy type of the iterated loop space $\Omega^{n} G$ of the gauge group $G[5,6]$. In discussing gauge invariance, one usually restricts to $\mathbf{G}$ which, being slightly smaller than the full group, gives the well defined topological properties just mentioned. These properties give rise, in particular, to isomorphisms among the homotopy groups, $\pi_{i+1}(\mathbf{A} / \mathbf{G}) \cong \pi_{i}(\mathbf{G})$ $\cong \pi_{i+n}(G)$. Non-vanishing of the homotopy groups implies non-triviality of the 
principal bundle $P: \mathbf{A} \rightarrow \mathbf{A} / \mathbf{G}$. This is the mathematical statement of the Gribov ambiguity since a non-trivial principal bundle has no global section. Typically, $G=S U(N)$. For $N$ large enough, Bott periodicity says that $\pi_{\text {even }}(G) \cong 0$, $\pi_{\text {odd }}(G) \cong \mathbb{Z}$.

On an even dimensional spin manifold, we denote the spin bundle of chirality \pm by $S^{ \pm}$. In a chiral gauge theory, the chiral Dirac operator $-i D_{ \pm}=-i \sigma_{ \pm} \cdot\left(\partial+\Gamma_{ \pm}+A\right)$ maps + spinors to - spinors, $-i D_{ \pm}$ $\in \operatorname{HOM}\left[\Gamma\left(S^{ \pm} \otimes E\right), \Gamma\left(S^{\mp} \otimes E\right)\right]$, where $E$ is a vector bundle in a certain representation of the gauge group. To study the gauge invariance of the quantum theory, one examines the vacuum functional of the chiral fermion, which is formally defined as the determinant of the chiral Dirac operator. Because of the gauge covariance of the chiral Dirac operator, the determinant can be thought of as a section of a line bundle on the orbit space. Whether the functional is gauge invariant depends on whether the line bundle is trivial. Does the topology of $\mathbf{A} / \mathbf{G}$ allow non-trivial line bundles? Complex line bundles are classified by the classes in the second cohomology of the base space. Typically, $\pi_{2}(\mathbf{A} / \mathbf{G}) \cong \mathbb{Z}, \pi_{1}(\mathbf{A} / \mathbf{G}) \cong 0$. By the Hurewicz isomorphism and the relation between homology and cohomology groups, $H^{2}(\mathbf{A} / \mathbf{G}) \cong \mathbb{Z}$. Hence line bundles are classified by these integers. The determinant can be a monopole wave function on a non-contractible sphere in A/G. The Atiyah-Singer index theorem provides the link between this possible non-triviality and the actual non-triviality of the determinant line bundle. The result in Atiyah and Singer [4] is that the determinant of the chiral Dirac operator $-i D_{ \pm}$corresponds to \pm 1 in $H^{2}(\mathbf{A} / \mathbf{G})$. We will see in Sect. 3 how the determinant actually behaves in an explicit example, and how this behavior is dictated by the presence of zero modes.

When there is a real structure [7], the determinant is a section of a real line bundle. Real line bundles are classified by the first Stiefel-Whitney class $w_{1} \in H^{1}(\mathbf{A} / \mathbf{G}, \mathbb{Z} / 2)$. $w_{1}$ not being zero will show up as a non-perturbative anomaly [7], which is associated with a Möbius strip like structure on a circle in $\mathbf{A} / \mathbf{G}$. In fact, non-perturbative anomalies also exist in odd dimensional space-time (in which there is no chirality). For $S U(2)$ gauge group in 3 dimensions, $H^{1}(\mathbf{A} / \mathbf{G}, \mathbb{Z} / 2)$ $\cong \mathbb{Z} / 2$. The Atiyah-Singer index theorem in Real $K$-theory predicts the nontriviality of the determinant line bundle.

All such anomalies can be discussed in the framework of canonical quantization [8-10]. In the $A_{0}=0$ gauge and static (or adiabatic) $\vec{A}$ background, one quantizes the fermion fields by solving the eigenvalues and eigenfunctions of the single particle chiral Dirac Hamiltonian,

$$
H \psi_{E}=E \psi_{E}, \quad H=-\overrightarrow{i \sigma} \cdot(\vec{\partial}+\vec{A}+\vec{\Gamma}),
$$

for one chirality. The spatial dimensions are compactified to $S^{n-1}$. A now is the space of all static gauge potentials with only spatial components and $\mathbf{G}$ is the group of time independent gauge transformations with a base point. All the general properties discussed earlier still apply when the dimensions are shifted by 1. Let $\Psi_{ \pm}$be the space spanned by the eigenfunctions of positive and negative energies. On a fixed fiber of $P: \mathbf{A} \rightarrow \mathbf{A} / \mathbf{G}$, the space $\Psi_{+} \oplus \Psi_{-}$is independent of the background (the gauge transformations) since the eigenvectors of different 
background span the same Hilbert space. However, in second quantization the Fock space is $\Lambda\left(\Psi_{+} \oplus \widetilde{\Psi}_{-}\right)=\Lambda\left(\Psi_{+}\right) \otimes \Lambda\left(\widetilde{\Psi}_{-}\right)$, where $\Lambda$ denotes the exterior algebra. For a Hermitian vector space $V, \Lambda(V)$ is isomorphic to $\Lambda(\tilde{V})$ up to a phase. In the familiar group theoretic language, the sum of all column Young diagrams is equal to the sum of all the complementary columns in the representations of $S U(V)$, modulo a $U(1)$ phase. Up to a phase, the isomorphism is, of course, given by the Levi-Civita $\varepsilon$-symbol. For a field theory, we have an infinite dimensional vector space and we need some form of regularization before we can attempt at identifying any such isomorphism. Physically, this isomorphism is simply the identification of holes in the filled Dirac sea to antiparticles. Before we make the identification, however, the afore-mentioned phase has to be looked at carefully. If the phase can be removed, we have a true isomorphism. The Fock space is then independent of the background. Quite generally, continuity requirements in the total space $A$ relate the phases at different fibers. As the fiber changes the spectrum also changes, and non-trivial phases can result from the interaction of positive and negative energy levels. For $\pi_{2}(\mathbf{G}) \cong \mathbb{Z}$, this can happen. The Atiyah-Singer index theorem predicts when these non-trivial phases actually occur. We will see in Sect. 4 how this happens explicitly. This phase gives rise to a 2-cocycle in the projective representation of the gauge group. In this paper, we are only interested in exhibiting the topological features of the cocycles. The algebraic properties have been studied in numerous other works [2].

For real cases, there is a symmetry between the positive and negative energies. $\Psi_{+}$and $\Psi_{-}$would be independent of the background if there were no overlap between them at all. They both would span a fixed Hilbert space. The Fock space $\Lambda\left(\Psi_{+}\right)$would be independent of the background. However, when a zero mode appears, a sign ambiguity arises in the identification of Fock spaces with a constant space. In Sect. 5, we will see how the sign enters the picture as a result of the spectral flow. A $\mathbb{Z} / 2$ extension of the group is needed. The non-trivial sign again is predicted by the index theorem.

\section{An Exact Fermion Determinant}

In this section, we solve the eigenvalue problem and the determinant of the chiral Dirac operator on a two dimensional spherical surface in the background of a nontrivial family of $S U(2)$ gauge potentials. Actually, the equations we are going to write down are slightly more general. It includes the chiral case, and the non-chiral case which will be useful in Sect. 5. The family is constructed as follows. Let $\mathbf{A}$ be the vector space of all $S U(2)$ gauge potentials on the spherical surface, $\mathbf{G}$ be the group of gauge transformations equal to the identity at the north pole. $\mathbf{A} / \mathbf{G}$ is a classifying space of $\mathbf{G}$. Now $\pi_{1}(\mathbf{G}) \cong \pi_{3}(S U(2)) \cong \mathbb{Z}$. We choose a representative of the generator of $\pi_{1}(\mathbf{G})$ as follows,

$$
g_{s}=\left[\begin{array}{cc}
e^{-i s / 2} & 0 \\
0 & e^{i s / 2}
\end{array}\right]\left[\begin{array}{cc}
\cos \frac{s}{2}+i \sin \frac{s}{2} \cos \theta & \sin \frac{s}{2} \sin \theta e^{i \varphi} \\
-\sin \frac{s}{2} \sin \theta e^{-i \varphi} & \cos \frac{s}{2}-i \sin \frac{s}{2} \cos \theta
\end{array}\right]
$$


which is periodic in $s$. It comes from the standard identification: $S^{3} \rightarrow S U(2)$. Here, $\theta, \varphi$ are the spherical coordinates.

We consider the class of potentials related to $A=0$ by gauge transformations generated by this matrix

$$
A_{\mathrm{s}}=g_{\mathrm{s}}^{-1} d g_{\mathrm{s}} \text {. }
$$

While this loop is not contractible in $\mathbf{G}$ at $[A=0]$, it is contractible in $\mathbf{A}$ to any point $A_{0}$. Let us arbitrarily take the point $A_{0}$ to be

$$
A_{0}=\left[\begin{array}{cc}
-i \sin \theta & e^{i \varphi} \cos \theta \\
-e^{-i \varphi} \cos \theta & i \sin \theta
\end{array}\right] d \theta+\left[\begin{array}{cc}
0 & i e^{i \varphi} \\
-i e^{-i \varphi} & 0
\end{array}\right] \sin \theta d \varphi .
$$

The family of $S U(2)$ gauge connections we are going to use is $\{A(s, t)$ $\left.=(1-t) A_{0}+t A_{s} \mid t \in[0,1], s \in[0,2 \pi]\right\}$, which is a disc in $\mathbf{A}$ with the boundary in the fiber of $P: \mathbf{A} \rightarrow \mathbf{A} / \mathbf{G}$ at $[A=0]$. $P$ projects $\{A(s, t)\}$ to a representative of the generator of $\pi_{2}(\mathbf{A} / \mathbf{G})$. Explicitly,

$$
\begin{aligned}
i A(s, t)= & d \theta\left[\begin{array}{cc}
p_{1} \sin \theta & e^{i \varphi}\left(p_{2}+i p_{1} \cos \theta\right) \\
e^{-i \varphi}\left(p_{2}-i p_{1} \cos \theta\right) & -p_{1} \sin \theta
\end{array}\right] \\
& +\sin \theta d \varphi\left[\begin{array}{cc}
p_{2} \sin \theta & -e^{i \varphi}\left(p_{1}-i p_{2} \cos \theta\right) \\
-e^{-i \varphi}\left(p_{1}+i p_{2} \cos \theta\right) & -p_{2} \sin \theta
\end{array}\right],
\end{aligned}
$$

where $p_{1}=\frac{t}{2} \sin s+(1-t), p_{2}=\frac{t}{2}(1-\cos s)$.

On the sphere we choose the metric

$$
d s^{2}=d \theta^{2}+\sin ^{2} \theta d \varphi^{2} .
$$

Since the tangent bundle is non-trivial, unit tangent and cotangent vectors are not globally defined. Let $N$ be the path $0 \leqq \theta<\pi$, and $S, 0<\theta \leqq \pi$. A set of orthonormal basis of the cotangent vectors is

$$
\left[\begin{array}{l}
e^{1} \\
e^{2}
\end{array}\right]^{N, S}=\left[\begin{array}{cc}
\cos \varphi & \delta^{N, S} \sin \varphi \\
-\delta^{N, S} \sin \varphi & \cos \varphi
\end{array}\right]\left[\begin{array}{c}
d \theta \\
\sin \theta d \varphi
\end{array}\right],
$$

where $\delta^{N}=-1, \delta^{s}=+1$. The patching functions and the connection are the following,

$$
\begin{gathered}
{\left[\begin{array}{l}
e^{1} \\
e^{2}
\end{array}\right]^{N}=\left[\begin{array}{c}
\cos 2 \varphi-\sin 2 \varphi \\
\sin 2 \varphi \cos 2 \varphi
\end{array}\right]\left[\begin{array}{l}
e^{1} \\
e^{2}
\end{array}\right]^{S},} \\
d\left[\begin{array}{l}
e^{1} \\
e^{2}
\end{array}\right]^{N, S}=\left[\begin{array}{rr}
0 & 1 \\
-1 & 0
\end{array}\right] \omega^{N, S}\left[\begin{array}{l}
e^{1} \\
e^{2}
\end{array}\right]^{N, S}, \quad \omega^{N, S}=\left(\delta^{N, S}+\cos \theta\right) d \varphi .
\end{gathered}
$$

The tangent vector basis is then given by

$$
\left[e_{1}, e_{2}\right]^{N, S}=\left[\partial_{\theta}, \frac{1}{\sin \theta} \partial_{\varphi}\right]\left[\begin{array}{cc}
\cos \varphi & \delta^{N, S} \sin \varphi \\
-\delta^{N, S} \sin \varphi & \sin \varphi \cos \varphi
\end{array}\right] \text { (no differentiation). }
$$


The spinors also have to be defined on $N$ and $S$, respectively,

$$
\psi_{ \pm}^{N}=e^{ \pm i \varphi} \psi_{ \pm}^{S}
$$

where \pm refers to the chirality. The connections for the spinors are

$$
\Gamma_{ \pm}^{N, S}= \pm \frac{i}{2} \omega^{N, S}= \pm \frac{i}{2}\left(\delta^{N, s}+\cos \theta\right) d \varphi .
$$

The chiral Dirac operator can now be written down,

$$
-i D_{ \pm}^{N, S}(A)=-i \sigma_{ \pm}^{N, S} \cdot\left(d P+\Gamma_{ \pm}^{N, S}+A\right),
$$

where $\sigma_{ \pm}^{N, S}= \pm i e_{1}^{N, S}+e_{2}^{N, S}, d P=\left(e_{1} e^{1}+e_{2} e^{2}\right)$. This operator maps + spinors to spinors, and vice versa,

$$
\chi_{\mp}^{N, S}=-i D_{ \pm}^{N, S} \psi_{ \pm}^{N, S} .
$$

We can eliminate the patching of $N$ and $S$ by the substitutions,

$$
\begin{gathered}
\chi_{\mp}^{N, S}=e^{ \pm i \delta^{N}, s_{\varphi / 2}} \chi_{\mp}, \quad \psi_{ \pm}^{N, S}=e^{\mp i \delta^{N}, s_{\varphi / 2}} \psi_{ \pm}, \\
-i D_{ \pm}^{N, S}=e^{ \pm i \delta^{N}, s_{\varphi / 2}}\left(-i D_{ \pm}\right) e^{ \pm i \delta^{N}, s_{\varphi / 2}}, \quad \chi_{\mp}=-i D_{ \pm_{\sharp}} \psi_{ \pm} .
\end{gathered}
$$

However, we have to be careful about the boundary conditions, e.g.,

$$
\chi_{ \pm}(\varphi+2 \pi, \theta)=-\chi_{ \pm}(\varphi, \theta) .
$$

For the connection $A(s, t)$, the differential operator is

$$
\begin{aligned}
-i D_{ \pm}[A(s, t)]= & \pm \partial_{\theta}-\frac{i}{\sin \theta} \partial_{\varphi} \pm \frac{1}{2} \cot \theta \\
& -p_{ \pm}\left[\begin{array}{cc}
\sin \theta & i e^{i \varphi}( \pm 1+\cos \theta) \\
i e^{-i \varphi}( \pm 1-\cos \theta) & -\sin \theta
\end{array}\right],
\end{aligned}
$$

where $p_{ \pm}= \pm i p_{1}+p_{2}($ see (3.4)).

The eigenvalue equation becomes finally

$$
-D_{ \pm}\left(p^{\prime}\right) D_{\mp}(p) \chi_{\mp}=\lambda_{\mp}\left(p^{\prime}, p\right) \chi_{\mp}
$$

where $p^{\prime}$ can be thought of as fixed. $-i D_{ \pm}\left(p^{\prime}\right)$ is needed to bring the spinor back to its original chirality after being acted on by $-i D_{\mp}(p)$. The eigenvalues are found by first factoring the equations and finding the series solutions. The characteristic values are fixed by comparing with the $A=0$ case, and the termination of the series. The eigenvalues are described as follows. The eigenvalues are labelled by $(m, n)$, $m=0, \pm 1, \pm 2, \ldots, n=0,1,2,3, \ldots$. For $m=n=0$,

$$
\lambda_{0}=\left(1-2 p^{\prime}{ }_{ \pm}\right)\left(1-2 p_{\mp}\right) .
$$

For $m=0, n=1,2,3, \ldots$, there is a pair of eigenvalues which are the roots of the quadratic equation,

$$
\left|\begin{array}{cc}
\lambda+(n+1)\left(-n-1+2 p_{\mp}\right)-2 p^{\prime}{ }_{ \pm}\left(2 p_{\mp}-1\right) & 2 p_{\mp}(n+1)-2 p^{\prime}{ }_{ \pm}\left(n+2 p_{\mp}\right) \\
-n\left(-1+2 p_{\mp}\right) & \lambda-\left(n+2 p_{\mp}\right) n
\end{array}\right|=0 .
$$


Note that the product of the two roots is

$$
\left|\begin{array}{cc}
(n+1)\left(-n-1+2 p_{\mp}\right)-2 p^{\prime}{ }_{ \pm}\left(2 p_{\mp}-1\right) & 2 p_{\mp}(n+1)-2 p^{\prime}{ }_{ \pm}\left(n+2 p_{\mp}\right) \\
-n\left(-1+2 p_{\mp}\right) & -\left(n+2 p_{\mp}\right) n
\end{array}\right|=n^{2}(1+n)^{2},
$$

which is independent of $p, p^{\prime}$. For $m \neq 0$, there is also a quadratic equation and the product of the two roots is again independent of $p, p^{\prime},(n+|m|-1)^{2}(n+|m|)^{2}$. These results show that there is no zero mode except for $\lambda_{0}$. By taking advantage of the fact that the products of the paired eigenvalues are constant, we can regularize the determinant in a simple way. Formally, the determinant is equal to the product of all eigenvalues up to a multiplicative constant. We can first take the products of the pairs and factor out these constants. Therefore, we get, in this simple regularization, just $\lambda_{0}$. In the chiral theory, $p^{\prime}$ can be taken to be a constant. Hence, we find that

$$
\operatorname{Det}\left[-i D_{ \pm}(p)\right]=1-2 p_{ \pm}=(1-t)(1 \pm 2 i)+t e^{ \pm i s} \text {. }
$$

At $t=1$, this is simply $e^{ \pm i s}$, which is a gauge dependent phase. The phase winds around the loop $\left\{A_{s}\right\}$ with winding number \pm 1 , respectively. In Fig. 1 we plot the contours of constant phase, and from these it is easily seen the ambiguity in the determinant originates from the zero mode located at

$$
(t \cos s, t \sin s)=\left(-\frac{1}{1+\sqrt{5}},-\frac{2}{1+\sqrt{5}}\right) .
$$

From the point of view of the gauge orbit space $\mathbf{A} / \mathbf{G}$, the boundary is identified as a point, and the determinant is seen to be a monopole wave function with a "string singularity" at that point.

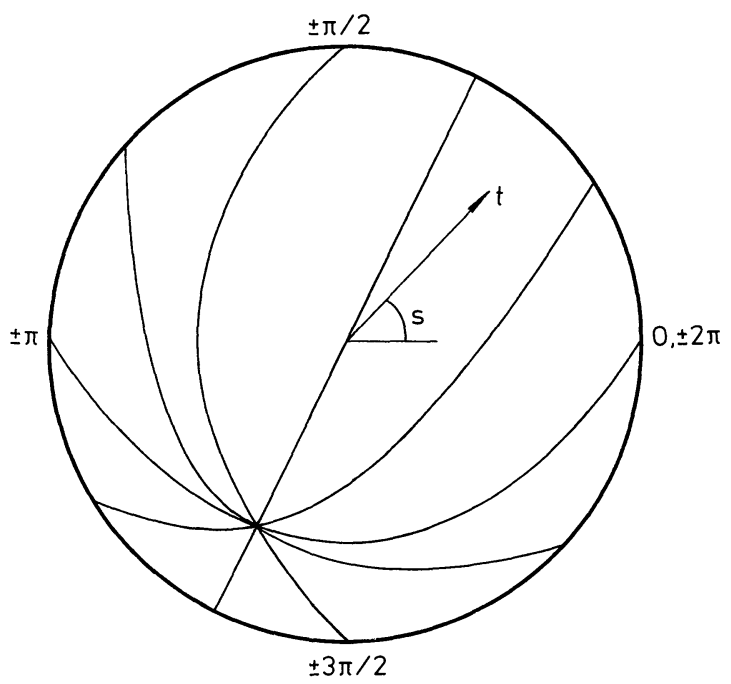

Fig. 1. Equal-phase curves for the fermion determinant 
The fermion determinant for the non-chiral case is obtained by setting $p^{\prime} \equiv p$,

$$
\operatorname{Det}(-i D)=\lambda_{0}(p, p)=5(1-t)^{2}+t^{2}+2 t(1-t)(\cos s+2 \sin s),
$$

which is equal to Det + . Det _. At the boundary, this is equal to 1 , which is certainly gauge independent.

\section{The Anomaly and the Dirac Hamiltonian}

The anomaly in Sect. 3 can also be studied by discussing the theory in terms of its Hamiltonian. In this context, the anomaly is manifested as a breakdown in the usual second quantization scheme in that we cannot find a basis consistent with gauge invariance everywhere.

We consider $A_{0}=0$ gauge, and $A_{x}$ being static (or adiabatically dependent on time). The one spatial dimension is compactified to a circle. We consider a representative of the generator of $\pi_{2}(\mathbf{G})$ given by

$$
g(\theta, \varphi)=\left[\begin{array}{cc}
e^{-i x / 2} & 0 \\
0 & e^{i x / 2}
\end{array}\right]\left[\begin{array}{cc}
\cos \frac{x}{2}+i \sin \frac{x}{2} \cos \theta & \sin \frac{x}{2} \sin \theta e^{i \varphi} \\
-\sin \frac{x}{2} \sin \theta e^{-i \varphi} & \cos \frac{x}{2}-i \sin \frac{x}{2} \cos \theta
\end{array}\right],
$$

where $\theta, \varphi$ parametrize the 2 -sphere, and $x$ is the spatial coordinate. This gives rise to the following representative of the generator of $\pi_{3}(\mathbf{G} / \mathbf{A})$,

$$
A=t g d g^{-1}=t \frac{i}{2} d x\left[\begin{array}{cc}
1-\cos \theta & i \sin \theta e^{-i(x-\varphi)} \\
-i \sin \theta e^{i(x-\varphi)} & -1+\cos \theta
\end{array}\right] .
$$

We take the connection for the spinors to be $i / 2 d x$, which is equivalent to taking a non-trivial spin structure on the circle. The single particle Hamiltonian for a chiral fermion, say of negative chirality, is then given by

$$
\begin{aligned}
H & =-i\left(\frac{d}{d x}+\frac{i}{2}+A_{x}\right) \\
& =-i \frac{d}{d x}+\frac{1}{2}+t \sin \frac{\theta}{2}\left[\begin{array}{cc}
\sin \frac{\theta}{2} & i \cos \frac{\theta}{2} e^{-i(x-\varphi)} \\
-i \cos \frac{\theta}{2} e^{i(x-\varphi)} & -\sin \frac{\theta}{2}
\end{array}\right] .
\end{aligned}
$$

The eigenfunctions are of the form

$$
\Psi_{n}=\left[\begin{array}{l}
a(\theta) e^{-i x} \\
b(\theta) e^{-i \varphi}
\end{array}\right] e^{i n x}
$$

and the eigenvalues are

$$
E_{n}^{ \pm}=n \pm \sqrt{\frac{1}{4}-t(1-t) \sin ^{2} \frac{\theta}{2}}
$$




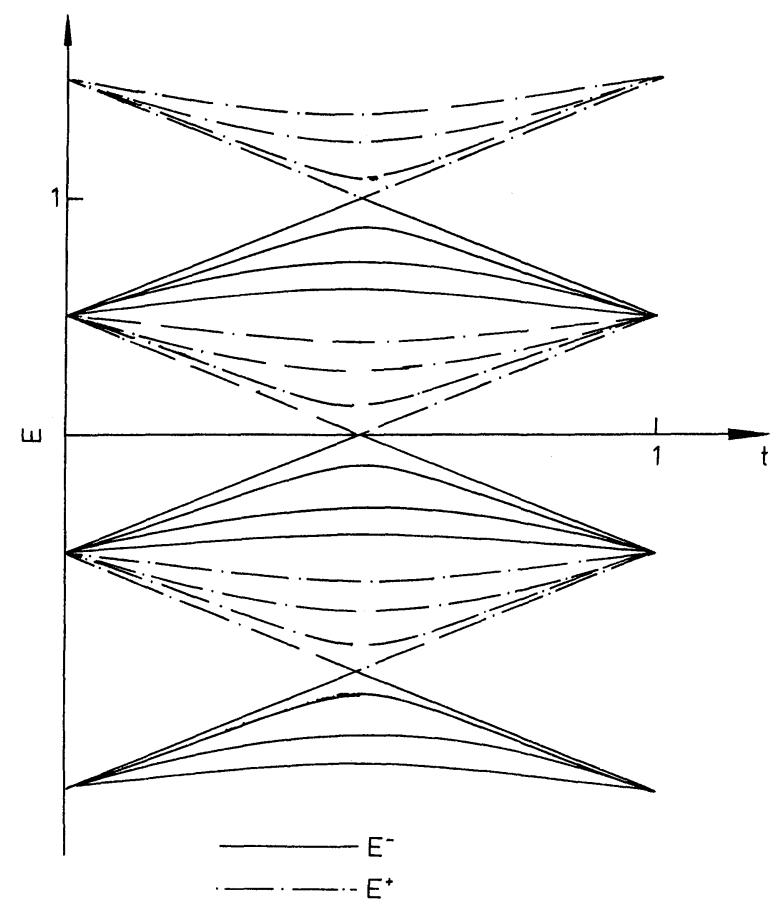

Fig. 2. Spectral flow diagram for the $1+1$ dimensional Hamiltonian

These eigenvalues form the pattern shown in Fig. 2. We focus our attention on $E_{0}^{ \pm}$and their wave functions. $E_{0}^{+}$and $E_{0}^{-}$have a zero mode at $t=1 / 2$ and $\theta=\pi$, where they become degenerate. Although other eigenvalues also have similar degeneracies, they have no zero modes. For the special eigenvalues, the wave functions are, respectively, at $t=0$,

$$
\left[\begin{array}{l}
0 \\
1
\end{array}\right] \text { and }\left[\begin{array}{c}
e^{-i x} \\
0
\end{array}\right],
$$

while at $t=1$, they are, respectively,

$$
\left[\begin{array}{c}
i \sin \frac{\theta}{2} e^{-i x} e^{i \varphi} \\
\cos \frac{\theta}{2}
\end{array}\right] \text { and }\left[\begin{array}{c}
-i \cos \frac{\theta}{2} e^{-i x} \\
\sin \frac{\theta}{2} e^{-i \varphi}
\end{array}\right] .
$$

These two wave functions are not well defined in the parameter space at $\theta=\pi$ because they depend on $\varphi$ at that point. They cannot be made well defined on the 2 -sphere in G. Indeed they are actually sections of the principal Hopf bundle. To get around this difficulty, one usually defines the functions on patches similar to 
those in Sect. 3

$$
\begin{gathered}
\Psi_{+}^{N}=\left[\begin{array}{c}
i \sin \frac{\theta}{2} e^{i \varphi} e^{-i x} \\
\cos \frac{\theta}{2}
\end{array}\right], \quad \Psi_{+}^{S}=\left[\begin{array}{c}
i \sin \frac{\theta}{2} e^{-i x} \\
\cos \frac{\theta}{2} e^{-i \varphi}
\end{array}\right], \quad e^{i \varphi} \Psi_{+}^{N}=\Psi_{+}^{S}, \\
\Psi_{-}^{N}=\left[\begin{array}{c}
-i \cos \frac{\theta}{2} e^{-i x} \\
\sin \frac{\theta}{2} e^{-i \varphi}
\end{array}\right], \quad \Psi_{-}^{S}=\left[\begin{array}{c}
-i \cos \frac{\theta}{2} e^{i \varphi} e^{-i x} \\
\sin \frac{\theta}{2}
\end{array}\right], \quad e^{-i \varphi} \Psi_{-}^{N}=\Psi_{+}^{S} .
\end{gathered}
$$

Note that the winding numbers of the patching functions are +1 and -1 , respectively. If we use these two wave functions to span a two dimensional space, the "monopole charges" will cancel and one can choose a new basis without the phase problem, e.g., one can choose

$$
\begin{aligned}
& \Psi_{1}=\cos \frac{\theta}{2} \Psi_{+}^{N}+\sin \frac{\theta}{2} \Psi_{-}^{S}=\left[\begin{array}{l}
0 \\
1
\end{array}\right] \\
& \Psi_{2}=-\sin \frac{\theta}{2} \Psi_{+}^{S}+\cos \frac{\theta}{2} \Psi_{-}^{N}=\left[\begin{array}{c}
-i e^{-i x} \\
0
\end{array}\right] .
\end{aligned}
$$

However, when we second quantize, the wave functions that go into the Fock space are $\Psi_{+}$and $\widetilde{\Psi}_{-}$, and this complex conjugation introduces a difficulty. The point is that now both functions have topological index +1 . If one insists on writing down an equation similar to (4.9), then $\Psi_{1}, \Psi_{2}$ will no longer be linearly independent on the whole 2 -sphere, and therefore, they cannot serve as a basis. It is easy to show that the transition from zero topological indices at $t=0$ to non-zero indices at $t=1$ occurs precisely at $t=1 / 2$, where there is a degeneracy. Although the topological charge is generally conserved at such degeneracies, second quantization introduces a twist in the space of fermion states at the zero mode degeneracy, and this twist makes it impossible to define a consistent basis. The other degeneracies cause no problems because taking the direct sum will always cancel the indices. Notice we have ignored the degeneracies at $\theta=0$. These degeneracies do not play a role in our discussions, and indeed, we can choose a simple perturbation to lift the degeneracies there without disturbing the topological features.

\section{The Non-Perturbative Anomaly in $2+1$ Dimensions}

Non-perturbative anomalies cannot be expressed as non-conservations of local currents, unlike the anomalies discussed in the previous two sections. In this section, we show how these anomalies arise explicitly, in a way similar to that disćussed in Sect. 4.

From the Hamiltonian point of view, if we consider a static background field in $2+1$ dimensions, we need to solve the equations of some Euclidean theory in 2 
dimensions. Once again we work in the $A_{0}=0$ gauge, and consider a family of gauge field background which has the same form as that in Sect. 3. The single particle Hamiltonian is then a (non-chiral) Dirac operator in 2 dimensions,

$$
H=\left[\begin{array}{cc}
0 & -i D_{-}(p) \\
-i D_{+}(p) & 0
\end{array}\right]
$$

The equation

$$
H \Psi=E \Psi, \quad \Psi=\frac{1}{\sqrt{2}}\left[\begin{array}{l}
\psi_{+} \\
\psi_{-}
\end{array}\right]
$$

is equivalent to

$$
-D_{+}(p) D_{-}(p) \psi_{-}=E^{2} \psi_{-}, \quad \psi_{+}=-\frac{i}{E} D_{-} \psi_{-} .
$$

We recognize immediately this equation is a special case of (3.16) with $p^{\prime}=p$, $E= \pm \sqrt{\lambda}$. For $p^{\prime}=p, \lambda$ 's are non-negative. Actually, the spinors we consider here have a real structure. We can find a basis in which both $H$ and $\Psi$ are completely real. We now pay special attention to the eigenstates with $E= \pm \sqrt{\lambda_{0}(p, p)}$. As $t$ goes from 0 to 1, $E$ changes in the fashion shown in Fig. 3. The different levels are

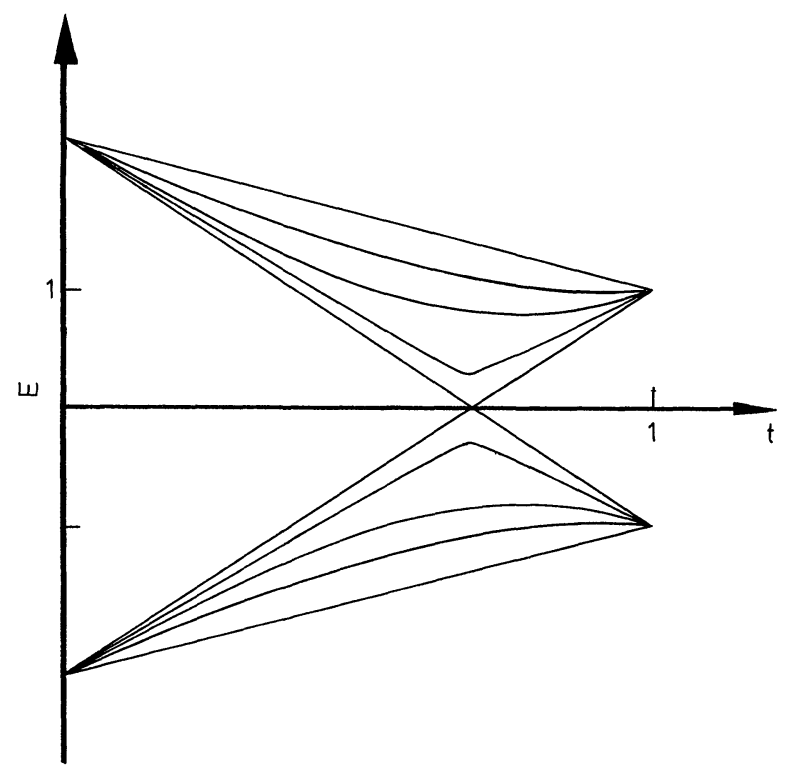

Fig. 3. Spectral flow diagram for the $1+2$ dimensional Hamiltonian 
for different values of $s$. The wave functions are

$$
\Psi^{N, S}=\frac{1}{\sqrt{8 \pi}}\left[\begin{array}{c} 
\pm e^{i \alpha-i \delta^{N, s_{\varphi / 2}}}\left[\begin{array}{c}
\sin \frac{\theta}{2} e^{i \varphi / 2} \\
-i \cos \frac{\theta}{2} e^{-i \varphi / 2}
\end{array}\right] \\
e^{i \delta^{N, s} s_{\varphi / 2}}\left[\begin{array}{c}
\cos \frac{\theta}{2} e^{i \varphi / 2} \\
i \sin \frac{\theta}{2} e^{-i \varphi / 2}
\end{array}\right]
\end{array}\right]
$$

where $\alpha$ is the phase of $\left[(1-t)(1-2 i)+t e^{-i s}\right]$. Now we use the unitary matrix

$$
U=\frac{1}{\sqrt{2}}\left[\begin{array}{rrrr}
1 & 0 & 0 & -1 \\
0 & 1 & 1 & 0 \\
0 & i & -i & 0 \\
-i & 0 & 0 & -i
\end{array}\right]
$$

and an overall phase to put the wave function into the real form. The result is

$$
\Psi^{N, S}=\frac{1}{2 \sqrt{\pi}}\left[\begin{array}{r}
-\sin \left(\frac{\alpha}{2}+\frac{1-\delta^{N, S}}{2} \varphi \mp \frac{\pi}{4}\right) \sin \frac{\theta}{2} \\
\cos \left(\frac{\alpha}{2}-\frac{1+\delta^{N, S}}{2} \varphi \mp \frac{\pi}{4}\right) \cos \frac{\theta}{2} \\
-\sin \left(\frac{\alpha}{2}-\frac{1+\delta^{N, S}}{2} \varphi \mp \frac{\pi}{4}\right) \cos \frac{\theta}{2} \\
\cos \left(\frac{\alpha}{2}+\frac{1-\delta^{N, S}}{2} \varphi \mp \frac{\pi}{4}\right) \sin \frac{\theta}{2}
\end{array}\right] .
$$

This wave function depends on $\alpha$ as a half angle. For each $t \in[0,1]$, as we let $s$ go from 0 to $2 \pi$, the wave function can change sign, depending on how $\alpha$ changes. From Fig. 1, it is clear that for $t<\sqrt{5} /(1+\sqrt{5})$, the change in $\alpha$ is zero while for $t>\sqrt{5} /(1+\sqrt{5})$ the change is $2 \pi$, and the wave function does change sign as $s$ is varied. We can follow the flow of eigenvalues as $t$ changes in terms of the possible changes in the eigenfunctions with the help of a topological index defined as follows. To each energy level of a real Hamiltonian that doesn't become degenerate when a parameter is varied, we assign a mod 2 index according to whether the wave function changes its sign. Should two levels touch each other as another parameter is varied, each level can acquire a new topological number upon their subsequent separation, but the sum will be conserved $(\bmod 2)$. For the case of a real fermi field, we need only consider the positive energy states in constructing the Fock space. At $t=0$, nothing depends on $s$. As $t$ increases, there may be exchanges of the topological number as a result of degeneracies, although the total is unchanged. This conservation fails, however, at $t=\sqrt{5} /(1+\sqrt{5})$ where the zero mode is 
situated, because a unit topological charge has escaped from the Fock space defined by positive energy states (see Fig. 3). Hence, for $t>\sqrt{5} /(1+\sqrt{5}$ ), the total index is $1 \bmod 2$. At $t=1$, in particular, there is a sign ambiguity around a noncontractible loop which cannot be eliminated by any change of basis. The fermion Fock space is therefore ill-defined as a representation of the gauge transformation group, and the theory is afflicted with a non-perturbative $\mathbb{Z} / 2$ anomaly. This anomaly was first discussed by Redlich [11] in the Euclidean functional integral formalism. An analogous situation in four dimensions was first discussed by Witten [7]. In that case, the Hamiltonian is of the form $i D$, where $D$ is a real skewadjoint operator.

\section{Conclusion}

We have given three explicit examples to show how topology and spectral flows can cause anomalies. In the first example, we were able to solve the exact spectrum and the determinant of the chiral Dirac operator in a non-trivial background. In the other two examples we showed how topology determines the spectral flow and how second quantization introduces a bundle twist in the fermion states. Zero modes are seen to be the origins of the topological twist in all three examples. Local properties of the zero modes in the gauge orbit space constrain the global properties of the quantum theory. Having these explicit examples can help elucidate the physical implications of anomalies, and other forms of topological obstructions [12].

After the completion of this work, we received a preprint [13] which contains essentially the same results as those in Sect. 3. $A_{0}$ is taken to be zero by the author instead of (3.3) in this paper.

Acknowledgements. We would like to thank O. Alvarez, R. E. Marshak, Y. Okamoto, F. Quinn, S. Rao, J. Slawny, G. Staebler, and C. Tze for discussions. This work was supported in part by NSF Grant 85-07170.

\section{References}

1. Zumino, B., Wu, Y.S., Zee, A.: Chiral anomalies, higher dimensions, and differential geometry. Nucl. Phys. B 239, 477-507 (1984)

2. Bardeen, W.A., White, A.R. (eds.): Symposium on anomalies, geometry, topology. Singapore: World Scientific 1985 and references therein

3. Alvarez-Gaumé, L., Ginsparg, P.: The topological meaning of nonabelian anomalies. Nucl. Phys. B 243, 449 (1984)

4. Atiyah, M., Singer, I.M.: Dirac operators coupled to vector potentials. Proc. Nat. Acad. Sci. USA 81, 2597 (1984)

5. Singer, I.M.: Some remarks on the Gribov ambiguity. Commun. Math. Phys. 60, 7-12 (1978)

6. Atiyah, M., Jones, J.D.: Topological aspects of Yang-Mills theory. Commun. Math. Phys. 61, 97-118 (1978)

7. Witten, E.: An $S U(2)$ anomaly. Phys. Lett. 117 B, 324 (1982)

8. Faddeev, L.D.: Operator anomaly for Gauss law. Phys. Lett. 145 B, 81 (1984)

9. Nelson, P., Alvarez-Gaumé, L.: Hamiltonian interpretation of anomalies. Commun. Math. Phys. 99, 103-114 (1985) 
10. Segal, G.: Faddeev's anomaly and Gauss' law, preprint, Oxford

11. Redlich, A.N.: Gauge noninvariance and parity nonconservation of three-dimensional fermions. Phys. Rev. Lett. 52, 18-21 (1984); Parity violation and gauge noninvariance of the effective gauge field action in three dimensions. Phys. Rev. D 29, 2366-2374 (1984)

12. Chang, L.N., Liang, Y.: To appear

13. Forte, S.: Two- and four-dimensional anomalies with an instanton background, preprint, MIT, CTP 1342; Phys. Lett. 174B, 309-312 (1986)

Communicated by L. Alvarez-Gaumé

Received June 24, 1986 